\title{
OBSERVATIONS
}

ON WHE

\section{TREATMENT OF SYPHILIS,}

wrru

AN ACCOUNT

op

SEVERAL CASES OF THAT DISEASE,

IN WHICH A CURE WAS EFFECTED WITHOUT THE USE OF MERCURY.

By THOMAS ROSE, EsQ. A.M.

OF BALLIOL COLLEGE, OXFORD; SURGEON TO THE SAINT JAMES'S IN" FIRMARY, AND TO THE COLDSTREAM REGIMENT OF GUARDS.

\section{Read June 24, 1817.}

THE existence of a numerous class of diseases, whose symptoms bear a close resemblance to those arising from the venereal poison, but which are curable without the exhibition of mercury, has for many years been satisfactorily ascertained, and is confirmed by the repeated experience of every surgeon. To form an acurate diagnosis between these and syphilis, is in many cases extremely perplexing; and the attention which has of late been bestowed on this important subject, has only shewn more clearly the difficulties in which it is involved. 
It unfortunately happens, that, unlike the effects of other morbific poisons, the symptoms of syphilis are not so marked and peculiar in their character, that by them alone the presence of its virus can be ascertained. Precisely similar ones occur in other diseases, and are even, in certain habits, induced by the action of mercury; and it is only in their history and progress, that any difference is to be detected. These are frequently so confused by the inaccuracy of patients, and other causes, that no reliance can be placed on them, and we shall find in practice, that there are few cases in which the nature of the disease is sufficiently decided, not to admit of some degree of doubt being entertained on the subject. Under such circumstances, we cannot wonder at the confused accounts which we meet with of this disease, and at the vague and discordant opinions which have at all times prevailed respecting it; nor, whilst its diagnosis rests on such obscure and unsatisfactory grounds, can we expect to find any consistent rules established for its treatment. Two points, however, appear to be generally agreed upon; namely, that syphilis does not admit of a natural cure, and that mercury is the only remedy hitherto known, which has the power of destroying its virus. So fully are these supposed to be established, that, where a disease has been cured without the use of that medicine, and has not afterwards returned, such fact alone, whatever may have been the symptoms, 
is regarded as sufficient proof that it was not a case of syphilis.

That mercury, where its action is properly regulated, and kept up for a sufficient time in the system, effectually destroys the venereal virus, is confirmed by the fullest experience. Its utility, both in the local and constitutional forms of the disease, is two obvious to admit of a question; but the absolute necessity for its employment is more diffcult of proof, and seems to have been assumed on less satisfactory evidence. It is true the majority of practitioners have concurred in this opinion during $\bar{a}$ long series of years; it may be said to be founded on the experience of more than three centuries. But this, although a strong argument in its favour, will not justify our yielding an implicit assent to a proposition so general and so important. The notions formerly entertained of this disease are known to have been extremely incorrect, and it should not be forgotten, that the same argument is not less conclusive, as to the necessity of a course of mercury in multitudes of cases which experience has now fully shewn to be even aggravated by its use.

The importance of this question is obvious, not so much in reference to the treatment of syphilis under common circumstances, for the strikingly good effects of mercury will probably not render it adviseable in general to give up the use of that re- 
medy, but from the change it will produce in our views of the diagnosis of the disease. The distinction which has engaged such a share of attention of late years, and which is evidently so important between syphilis and syphiloid diseases, has been made to depend so much on the former admitting of no cure except by mercury, that if this principle should be found to be erroneous, the difficulties which have attended it will in a great measure be explained. That it is erroneous I shall endeavour to prove, by shewing first, that it is irreconcileable with some general facts, and secondly, that it is contradicted by particular cases.

In venturing to lay before the Society, these objections to a doctrine so long and so universally maintained, I do not presume to state on such limited experience, that no cases of syphilis can occur in which mercury may be essentially necessary. Still less can I be misunderstood to suppose that it is not often highly beneficial, but I believe that our ideas on this subject have been carried beyond the truth, and that from perhaps the occasional necessity, and certainly the general utility of the remedy in such complaints, an unwarrantable inference has been drawn that they never can be cured without it.

First then it appears to me impossible to reconcile many well authenticated facts with such a theory. A few of these I shall briefly enumerate. 
1. Other specifics have been occasionally tried in the cure of this disease, at every period since it is considered as having made its first appearance, and the success of some of them has been attested, after ample experience, by men of veracity and of acknowledged abilities. It is highly probable that many of the cases supposed to establish the efficacy of these remedies were not venereal; and, from their uniformly having fallen into disuse, we may take it for granted, that the remedies themselves were not possessed of all the virtues attributed to them; but if no instance of syphilis can be cured without a course of mercury, can we possibly account for a single rational practitioner having been deceived by them? It has been said that the efficacy of these remedies in syphilis has been erroneously inferred from their being serviceable in removing the sequelæ of the disease, the direct effects of mercury and the cachexia syphiloidea; but this is by no means sufficient to explain the accounts given of them, by those who were so prejudiced in their favour, as to lay aside mercury, and trust to them in every case which they met with. Sarsaparilla, carbonate of ammonia, opium, the diffierent acids, and many other supposed specifics, have been tried on extensive scales, and the testimonies in their favour must astonish all who believe a cure without mercury to be impossible.

2.I n several parts of Europe, mercury is either not used at all, or administered in such a manner 
as we should consider totally inadequate to the cure of the disease.

I shall select only a single instance: in the Fourth Volume of the Transactions of this Societý, a curious account will be found by Mr. Fergusson, of the treatment of the venereal disease in Portugal. It appears, that the use of mercury in that complaint is there almost entirely abandoned, and the consequences have not been such, as, according to our ideas, might naturally have been expected. It is reasonable to think, that a considerable part of the cases of which Mr. Fergusson speaks, would not be regarded by cautious practitioners as venereal. In mentioning the effects of the disease on the British soldiers, he states, that "the constitution, while strongly under the influence of mercury, became affected with the secondary symptoms in a proportion that could not have been expected," and some other circumstances which would lead us to believe, that several of the cases must at least have been of a doubtful nature; but the fact itself; that mercury is hardly at all employed in that country for the cure of any such diseases, is cer. tain, not only from Mr. Fergusson's account, but from the observations of the numerous British surgeons who have served there; and when we reflect on the well known contagious properties of the syphilitic virus, we shall find it difficult to comprehend, why, when thus left to itself, it should 
not be productive of far more serious mischief* If we compare the account of this disease in Portugal, with what Mr. Hunter considered the natural result of its introduction, where the means of cure were not understood, or with the description

* I had several opportunities, in the course of the Peninsular war, of witnessing the little attention paid to this disease in its earlier stages, both by the Portuguese and Spaniards. I was able to trace some of the natives of those countries (who were attached to the commissariat), in perfect health for two or three years, after sores, which $I$ had supposed to be venereal, had been healed without mercury. The use of that remedy had been prevented both by their own unwillingness to have recourse to it, and by the almost constant exposure to the open air, both during the night and day, which the care of their mules required of them. A few similar instances came under my observation amongst our own soldiers, where the use of mercury was interrupted at early periods by movements of the army or other causes, and was not afterwards resumed. I have often wondered, that, in not one of these, any ill effects ensued; but I could only infer, that my opinion of the nature of the disease had been erroneous, although, in the cases to which I allude, it had by no means been hastily formed, and the sores had had every character of true chancres.

A few instances of nodes, and caries of different bones, are to be met with in Portugal, as in every part of the world; but they did not appear to me at all more frequent there, than in any other country I have had an opportunity of visiting. I examined some of those objects, but did not chance to meet with a single one, where the history of the disease corresponded with the idea of its being syphilis. It may be remarked, that although mercury is scarcely thought of in that country for the cure of primary sores, or of such cases where its virtues are undoubted, it is often hal. recourse to, very unmercifully, in those diseases in which its effects. are most pernicious.

voL. VIII.

A A 
of its ravages when it is imagined to have first shewn itself at the close of the 15th century, the contrast cannot fail to strike us. In endeavouring to prove that it was taken to the island of Otaheite, by some of the crew of $M$. Bougainville, and not by that of Captain Wallis, Mr. Hunter remarks, that, "6 as M. Bougainville arrived at the island ten months after Wallis, there was a sufficient time, if any one of the crew of the latter had been diseased, for the inhabitants of the whole island to have been infected; and the ravages of the disease must have been evident to the crew of Bougainville immediately upon their arrival." The accounts of the destruction occasioned by the venereal disease in Otaheite, appear by subsequent observations to be entirely unfounded; but they are referred to here, because, if the commonly received ideas on the subject are correct, such would certainly be the consequences of that poison being introduced into any country, where the sole antidote to it was either unknown or disregarded; whilst the wellauthenticated description of its mild effects in Portugal, when not interrupted by mercury, is totally irreconcileable with these ideas. Mr. Carmichael supposes, from the description given by Mr. Fergusson, that the disease which prevails in Portugal, is a phagedenic or sloughing ulcer, differing from the true syphilitic chancre, and in which he has not found mercury to be either necessary or serviceable ; but in what manner that kingdom, over-run by every description of foreigners, 
has escaped the introduction of syphilis itself, is left entirely to conjecture.

3. Mr. Fergusson mentions in the same paper a fact, which $I$ had also heard from others, that in the German regiments in our service, some surgeons " pertinaciously even officially refused to prescribe mercury in syphilis, asserting that it was not necessary to the cure." "Such alarming conduct," he adds, "as being referable only to the most brutal ignorance, of course met with no quarter." A similar circumstance was communicated to me by the late Dr. Banks, who had served some time in the Mediterranean. He assured me from his own observation, that the surgeon of one of our foreign regiments * to which he himself was attached, used no mercury for several years in venereal complaints, and believed that secondary symptoms did not occur except where that medicine was employed.

Lastly; I have tried the same system in the Coldstream regiment of Guards during the last year and three quarters, and have certainly succeeded in curing all the ulcers on the parts of generation, which I have met with in that period, with the constitutional symptoms to which they

* I believe he said the Maltese regiment; but I forget exactly which he named. Dr. Theod. Gordon perfectly recollects the conversation to which I allude. 
gave rise, without the exhibition of mercury. I may not be warranted in asserting that many of these were venereal, but undoubtedly a considerable number of them had all the appearances of the primary sores produced by the venereal virus, and arose under circumstances where there had been at least a possibility of that virus having been applied. Admitting that there is nothing so characteristic in a chancre, as to furnish incontrovertible proof of its nature, it will yet be allowed, that there are many symptoms common to such sores, although not entirely peculiar to them, and wherever these are met with, there are strong grounds to suspect that they are the effects of the syphilitic virus. In a sore, for instance, appearing shortly after suspicious connection, where there is loss of substance, a want of disposition to granulate, and an indurated margin and base, there is certainly a probability of that poison being present. Amongst a number of cases of such a description, taken indiscriminately, the probability of some being venereal, is materially increased, and must at last approach nearly to a certainty. On this principle, some of the sores here referred to must have been venereal. They were seen also by different surgeons on whose judgment I could rely*, who agreed in considering many of them as well marked cases

* They were under the constant superintendance of Mr. Simpson, and were seen by Mr. Brodie, Mr. Bacot, Mr. Worrell, and many others. 
of true chancre. The battalion of the Coldstream, in which they occurred, consisted of upwards of a thousand men, who, being stationed in this metropolis, and associating often with the lower orders of prostitutes, were particularly exposed to the risk of infection, and might have been expected, in a much shorter period, to furnish many examples of that disease. This fact is in itself curious, independently of the character of the sores, and we may at least conclude from it, that the cases which absolutely require mercury for their cure, are far more uncommon than any one has hitherto imagined. The peculiar advantages which the surgeon of a regiment has of watching his patients for any period of time he chuses, after their cure, renders the history of those which occurred in the Coldstream Hospital more satisfactory than it could have been in any of our public institutions. The men who had been treated on this system, were examined almost every week for a considerable time after their apparent cure, both that the first approach of constitutional symptoms might be observed, and that any deception from an underhand use of mercury might be guarded against.

Previously to adopting this system, I tried in many instances very short courses of mercury in venereal sores, such as would not generally be considered adequate to the cure of the disease. In many of these a considerable hardness of the cica- 
trix was left, which is always regarded as a suspicious circumstance, but no secondary symptoms followed. Two cases, during the year 1814, in which mercury was used for only fourteen days, producing but a very slight effect on the gums, and in each of which the sore had healed with hardness, returned afterwards with bubo: the one in six weeks, and the other in about four months, after discontinuing the mercury. Each had a single gland enlarged in his groin, which was dispersed in a few weeks by rest and cold applications, and both have since continued well. Meeting with complete success from this plan of treatment, and satisfied that the ideas I had previously entertained of the disease were erroneous, I ventured to lay aside mercury entirely, with a view of observing for a time the progress of the virus, when not interfered with by that specific. This we have seldom an opportunity of doing, as, from the confidence so universally entertained in it as an antidote to syphilis, few cases of the advanced stages of the disease are met with, in which mer. cury has not been administered in some form or other. The appearances of the sores were carefully noted, in hopes of determining more accurately, in the first instance, in what cases mercury was absolutely required, and with the view of inquiring afterwards, what quantity of that medicine would be sufficient. The result has been different from what I had expected, and the cure in every 
instance* has been effected without the necessity of having recourse to any mercury whatever. This was begun in the month of June, 1815, and has been continued till the end of February in the present year, when the battalion moved to Windsor.

I have also used no mercury in a number of cases of primary sores, which came under my care during the last sixteen months, in the Saint James's Infirmary; but on these I place less reliance, as a few of them only could be watched for any time after their cure, and it is possible some of them may have since gone into other hospitals. The same objections apply to the trial which was given to this method in the York Hospital, at Chelsea; the men having either returned to their regiments, or being discharged the service shortly after their cure. Dr. James Forbes, physician to that hospital, informs me that upwards of sixty cases of ulcers on the penis were cured there by simple dressings; under the care of Mr. Dease. "In none of them was any local stimulant or escharotic employed, and the only general remedy was an occasional purgative. One only bid defiance to this simple

* It is not worth mentioning as an exception to this, that two cases of inflamed iris, with eruptions, took calomel pills for a short period, and that in two cases of sores, neither of them at all serious ones, I gave a little corrosive sublimate for four or five days, to try its effects in hastening the cure. 
management*, and out of the whole discharged, one only returned with an excoriation on the site of the old chancre, which got well in a very short time. This man had buboes, but they remained well." This plan has been tried pretty extensively by my friend, Mr. Whymper, surgeon to the second battalion of the Coldstream, in France, without a single instance of failure during more than a twelvemonth. Mr. Good adopted the same system in the hospital of the second battalion of the third regiment of guards at Windsor, and found it completely to answer. Similar trials have been made in a great many other quarters, I believe with uniform success. Some of these may probably hereafter be communicated to this Society.

Many other general facts might be added to those above enumerated, which are equally inexplicable, if the venereal disease possesses those contagious properties which are usually attributed to it, and is curable only by one specific: with these, however, I shall not occupy the time of the Society; but shall beg leave,

Secondly, to lay before it a few cases of ulcers on the parts of generation, in which no mercury was employed, from which every one can form his own conclusions. It will not be necessary

* This man was put through a course of mercury in compliance with the opinion of another surgeon, and not from any other cause. 
to give a minute description of the treatment adopted. All ideas of specific remedies were entirely laid aside. The patients were usually confined to their beds, and such local applications were employed, as the appearances of the sores seemed to indicate. Aperient medicines, antimony, bark, vitriolic acid, and occasionally sarsaparilla, were administered, if from any circumstances they were judged necessary.

1st. Sores which were not followed by any constitutional affection.

\section{CASE I.}

John Moulsham was admitted into the Coldstream Hospital on the 26th of August, 1815, with a sore of the size of a split pea, where the prepuce joins the corona glandis. The sore was circular, without granulations, with matter adhering to its surface, and with a hard and thickened margin somewhat elevated. He had a similar sore on the outer edge of the prepuce. The sores had been present a fortnight, and came five or six days after suspicious connection.

A sedative lotion, composed of five grains of the extract of hemlock, and the same quantity of opium to an ounce of water, was ordered to be ap- 
plied to the sores every two or three hours. On the 31st of August the sores were dressed with Bates's camphorated lotion, and on the 5th of September with the red precipitate ointment.

September 10. The sores were healed, with a great deal of hardness round the cicatrix of the one first described.

September 21. He was dismissed, at which time the hardness had a good deal diminished.

\section{CASE II.}

John Johnson was admitted into the same hospital on the 27th of August, 1815, with a sore as large as a sixpence on the internal prepuce, which had destroyed the margin of the corona glandis. The surface was sloughy; the sore was not very deep; but was attended with a very great degree of induration. It came eight days before his admission, and two days after suspicious connection.

The treatment was the same as in the last case. On the 16th of September the sore was healed with a very hard cicatrix, to which a little mercurial ointment and camphor was applied. He was dismissed on the 25th of September; and on the 12th of November the hardness was found entirely gone. 


\section{CASE III.}

Richard Webb, admitted into the same hospital on the 11 th of September, with a large indolent sore on the external part of his prepuce, of fourteen days' duration. It came a few days after suspicious connection.

On the 3d of October the sore was healed, with a very hard and slightly elevated cicatrix. He was dismissed on the 6 th.

\section{CASE IV.}

James Moore was admitted as above, on the 11th of December, 1815, with a deep foul sore close to the corona glandis, with considerable hardness and thickening of its base and margin. He said it had been present a month, and that it came about a week after suspicious connection. It had occasioned a bubo in his right groin, which had suppurated and burst. He was just returned from a detached duty, and from the acrid discharge of the sore, the prepuce was inflamed and excoriated.

On the 4th of January, 1816, the sore was healed with considerable hardness, and on the 13th the bubo also was healed, and he was dismissed. The 
hardness of the cicatrix had somewhat diminished by the use of a little mercurial ointment and camphor, but was still very considerable.

\section{CASE V.}

The same man was re-admitted on the 21st of February, 1816, with a deep ulcer of the corona glandis of the size of a silver penny, and a smaller one immediately bordering on it. A portion of the glans was destroyed; the sores were highly inflamed, with a dark slough in their centres, and with hard and thickened margins. The whole prepuce was thickened and inflamed, and several small ulcers had formed from the acrid discharge. The sores had been present three weeks, and came a week after suspicious connection.

His general health was good, and the cicatrix of the old sore had nearly lost its hardness.

Equal parts of Bates's camphorated water, and the sedative lotion, were applied to the sores.

February 24. The two sores had run into one. The sloughs had separated.

February 29. The sore was deep, and as large 
as a shilling, without any disposition to form granulations.

It was dressed with Bates's camphorated lotion.

March 5. It had become sloughy, and was spreading. The compound tincture of Benzoin was applied.

March 9. A deep slough had separated, and the edges of the sore looked better.

To return to the former lotion.

March 26. The sore was healing favourably.

The red precipitate ointment was applied.

April 16. It was healed, with considerable loss of substance, and much hardness of the cicatrix, and on the 21st he was dismissed.

June 8. His health was perfectly good, but the cicatrix was still very hard.

October 6. The cicatrix was nearly natural.

CASE VI.

Joshua Reeves was admitted on the 24th of 
January, 1816, with a foul, irritable sore, having a thickened margin, where the internal prepuce joins the upper part of the corona glandis, to which it had contracted some preternatural adhesions. The sore was of eight days' standing, and came a week after suspicious connection.

On the 24th of February the sore was healed, with considerable thickening and hardness, and on the 28th he was dismissed.

\section{CASE VII.}

Thomas Benton was admitted on the 8th of June, 1816, with a sore which had eroded underneath the frænum, and had the common appearances of a chancre in that situation. There was considerable induration of the frænum, and margins of the ulcer. He said it had been present only a day or two, and arose from a suspicious connection a week before.

The frænum was destroyed by a ligature, and a solution of opium and hemlock applied.

June 20. The sore was healed with hardness, and he was dismissed.

June 26. He returned with an inflamed gland 
in his left groin, which suppurated; and burst on the 11 th of July. In the beginning of that month the cicatrix of the chancre inflamed and ulcerated. The sore proved tedious, and the hardness around it increased. On the 7 th of August both the bubo and this sore were healed; the latter with a great deal of hardness. He remained in the hospital till the $2 \mathrm{~d}$ of September, on account of an inflammation of one of the lacunæ immediately within the orifice of the urethra. The hardened cicatrix of the chancre had a good deal diminished by the use of some mercurial ointment and camphor.

October 6. His health had continued good, and the induration of the cicatrix had disappeared.

\section{CASE VIII.}

Robert Staunton, a seaman, twenty-three years of age, was admitted into the Saint James's Infirmary on the 13th of November, 1816, with two sores on the internal surface of the prepuce, one on each side of the frænum. The sores were circular, and each about two-tenths of an inch in diameter. They had a dark-coloured matter adhering to their surface, were attended with considerable loss of substance, and shewed no disposition to granulate. Their edges were irregular, and there was a very considerable induration of their margins 
and base. The prepuce was inflamed and tense. There was an incipient bubo in his left groin. He observed the sores a few days previous to his admission, and had been exposed to the risk of infection at Portsmouth about a week before.

November 22. The sores had gradually spread, and complete phymosis had come on. I found it necessary to slit up the prepuce, that the sores might be more carefully cleaned; a small artery on the divided surface began to bleed a few hours afterwards, and he lost eight or ten ounces of blood. It was stopt by a ligature.

The sores continued to spread for some time, and proved extremely irritable, occasioning much pain, and a good deal of disturbance of the system. They spread over the whole of the prepuce, and destroyed a small portion of the margin of the corona glandis. On the 20th of January they were healed with a good deal of hardness and thickening, and the bubo was dispersed.

He was dismissed in the end of January, but was seen frequently afterwards, and as late as May the 3rd: the hardness of the cicatrices gradually disappeared, and his general health continued very good.

Several cases occurred of a cluster of ill-conditioned sores over the whole inner surface of the 
prepuce, and behind the corona glandis ; and also, of a circle of small irritable sores, situated on a thickened and contracted ring at the extreme margin of the prepuce. These occasionally produced buboes. It is doubtful with many how far such cases should be regarded as venereal; a chancre being supposed to be, generally, a solitary ulcer. All which I met with of this description healed readily, and were in no instance followed by any constitutional affection.

In one case of phagedenic ulcers the whole of the glans penis was destroyed, and in another, a considerable portion of it. These cases I shall describe, as they were the only ones in which any degree of permanent deformity was produced. In both there was such disturbance of the system from the moment of their admission, that the use of mercury would have been highly improper. I have several times seen that medicine exhibited under such circumstances*, and it has always appeared to me to hurry on the ravages it was intended to check. The same remark applies to the early use of bark, wine, and any tonics or stimulants. I am not at all aware whether such sores

- I have at present a patient under my care in the Saint James's Infirmary, who had used mercury for eight days in such a description of sores. The whole glans was destroyed, and the disease was making rapid progress under that treatment. The greatest benefit was experienced from bleeding and a change of system.
voL. VIII.
B B 
are produced by the syphilitic poison or not. They are seldom followed by secondary symptoms; but this has been accounted for, by the parts contaminated being so rapidly destroyed. They appear at any rate to be occasioned by the application of some morbific matter, and it is not easy to explain, whether the great degree of erethismus excited by the local affection, should be attributed to any peculiarity in that matter, or is owing to the peculiar state of constitution of the person infected. Mr. Fergusson gives a case of this nature, in which he says, " the infection was communicated by an opera-dancer at Lisbon, apparently in perfect health, who continued on the stage for several months afterwards, occasionally infecting others, without any thing extraordinary, as far as he could learn, in the nature of the symptoms *."

\section{CASE IX.}

Thomas Clarke, of a full habit of body, twentyone years of age, was admitted into the Coldstream Hospital on the 28th of March, 1816, with six or eight deep irritable sores on his internal prepuce. The surface of these was covered with a darkcolored slough ; they had thickened and highly inflamed margins, and discharged a very acrid ichor.

- Medico-Chirurgical Transactions, Vol. IV. p. 12. 
He complained of much headach and thirst; had a quick pulse, and other febrile symptoms. The sores had been present three days, and were first perceived a week after a suspicious connection. There was a chain of enlarged glands in each groin, which he said was of long standing.

He was ordered a brisk dose of jalap and cream of tartar, and afterwards six grains of antimonial powder, with a small quantity of Epsom salts every four hours; cold saturnine lotion was applied to the parts.

On the following days the febrile symptoms were increased. He had restless nights, frequent cold chills, much headach, and a constant irritable cough. His tongue was covered with a white fur; his pulse was quick and not easily compressed, and his skin dry and hot. He had much pain in the sores, which were rapidly extending and running into one another; a dark-coloured inflammation surrounding them, which terminated immediately in gangrene.

He had been freely purged by his medicines.

The cold lotion was laid aside, and the parts were fomented with a decoction of poppy-heads.

March 31. The sloughing had extended, and a considerable portion of the centre of the corona в B 2 
glandis was destroyed. A hæmorrhage'took place from it this morning, by which he had lost a pint of blood before I saw him. An artery of some size in the glans was secured by ligature. Equal parts of balsam of sulphur and oil of turpentine were applied to the sore, and the cold lotion was again had recourse to.

April 1. The sloughs had no disposition to separate, and on the body of the glans, anterior to the margin of the sore, several dark-coloured spots had shewn themselves. He had violent burning pain in the glans; his face was flushed; his tongue covered with a brown fur, and his pulse 102.

April 2. He has had two returns of hæmorrhage, but not to a great extent. $\mathrm{He}$ is somewhat less feverish, but weaker. The prepuce was slit open ; the sore dressed with compound tincture of benzoin, and a fermenting poultice applied. He was allowed beef-tea, and ordered a draught of camphor mixture and spirits of ammonia every three hours, and some compound powder of ipecacuanha at night.

April 3. The burning pain was relieved, and there was a good deal of dark-coloured discharge in the poultice. The glans was separated from the corpora cavernosa, but the part which surrounded the urethra was still entire. His pulse was softer, and only 92. 
April 5. The sloughs had nearly separated, after the whole glans, and the part of the urethra which passes through it, were destroyed. The sore continued irritable and much disposed to slough, and there was a good deal of fever.

April 13. Healthy granulations have begun to shew themselves. The patient has half a pint of wine, and a generous diet.

May 24. Every thing has gone on very favourably, and the sore has at last healed. The chain of enlarged glans has subsided, and the patient has recovered his health and looks.

This man deserted soon after he was dismissed from the hospital. He was apprehended in a few months, and sent to a foreign corps, at which time he had no appearance of secondary symptoms.

Much benefit would probably have been derived from bleeding on his first admission, which the symptoms strongly indicated.

\section{CASE X.}

Ephraim Johnson, aged twenty-nine, was admitted into the Coldstream Hospital on the 27th of May, 1816, with partial phymosis. His pre- 
puce was swollen and much inflamed, and there was a most acrid and copious discharge from underneath it, excoriating the neighbouring parts. The whole margin of the corona and the upper surface of the glans were covered with small white vesications. He said he had observed a deep sore on the inner prepuce three days before his admission, and that he was exposed to the risk of infection on the night of the 20th.

He had cold chills, a violent head-ach, complained of much thirst, and had a hard and quick pulse.

He was bled to the extent of a pint and a half, and ordered a solution of Epsom salts, and three grains of antimonial powder, every four hours.

The solution of opium and hemlock was directed to be injected frequently within the prepuce, and to be applied round the penis.

May 28. Had passed a restless night, with much burning pain in the parts; a frequent irritable cough had come on, and his febrile symptoms continued. The prepuce was divided ; it was covered with deep irritable sores, having a very acrid discharge, and was much inflamed and thickened. The sores were so numerous as to give it completely a honeycomb appearance. 
His bowels had been opened but once; the blood was slightly cupped.

The bleeding was repeated to twenty ounces, and the medicines ordered to be continued.

May 29. He had less pain in the penis, but the sores were deeper and more numerous, both over the inner membrane of the prepuce, and over the surface of the glans. He had had two stools; complained less of head-ach and thirst, and his pulse was softer. The blood had a slight coat of buff. The cough was troublesome.

His medicines were continued, with ten grains of Dover's powder at bed-time, and a blister was applied to his chest.

May 30. His bowels had been freely opened, and the febrile symptoms were milder. The sores had extended, and were become deeper and more sloughy.

May 31. He had suffered much pain in the night, and the sloughing had gone on; a portion of the glans was destroyed, and it was separated from the right corpus cavernosum. He had still much cough, and some head-ach.

Compound tincture of benzoin was applied, but gave very severe pain for about half an hour. The 
following lotion was therefore substituted for it, and the penis was covered with linen dipped in decoction of poppies. He was allowed some beef-tea.

R Extracti opii,

Conii aā Эi.

Liq. Plumbi Acetat. 3ij.

Aq. Fontan. zij. M. fiat lotio.

2dâ. quâque horâ partibus affectis applicand.

June 1. He had slept well, and was easier. The sores looked better.

June 4. Had gone on well for the last day or two, and the sores had improved.

June 7. The disposition to slough was quite checked, but the ulcers were very irritable. Complained of pain in his left groin, where a gland had enlarged; was ordered some decoction of bark, with a little tincture of rhubarb, and a nourishing diet.

June 17. The sore was healthy.

July 6. It was healed, but the bubo had increased, and was slowly suppurating.

July 11. The bubo had burst in two places.

July 31. The sores on the groin were healed, 
but the glands continued larger than natural. The cicatrix on the penis was thickened. His general health was good; a mercurial plaster was applied to the groin, to be worn for some weeks, and he was dismissed.

The absorbent glands of the groin were affected in a great many cases, and often suppurated. The abscesses were in general allowed to burst of themselves, and almost all of them healed readily.

A very few exceptions to this were met with. In one case sloughing took place to an alarming extent. This occurred in March, 1816, in a recruit, who was admitted in a bad state of general health, with an open bubo covered by a good deal of diseased integuments. He had been using mercury internally for three weeks previous to his enlisting, on account of some suspicious sores on his penis. The skin forming the margins of the ulcer was attacked with a dark-coloured inflammation, running immediately into gangrene. The sore in this manner spread rapidly. Sloughing of the cellular membrane on the base of the ulcer, and of some diseased absorbent glands, also took place. There was great constitutional irritation and intense burning pain. The principal vessels were at last exposed, but the disease fortunately stopt, under the use of a cooling regimen, and of diaphoretic medicines. The sore afterwards healed 
very favourably, and the man has since continued well.

Another case of bubo proved troublesome, from the number of absorbent glands which became affected. This case I shall briefly detail.

\section{CASE XI.}

John Mabry was admitted into the Coldstream Hospital on the 24th of October, 1815, with a chancre, which came ten days after suspicious connection, and was healed with a great deal of hardness, by means of topical applictions, on the 24th of November. On the 1st of that month a gland inflamed in his right groin, which suppurated rapidly, and, as it occasioned a great deal of pain, it was opened with the lancet on the 13th of November.

January 2d, 1816. The bubo was not disposed to heal. There were several diseased glands close to each other, and the discharge was confined by discoloured and inflamed integuments. The cuticle had been abraded from the cicatrix of the sore on the penis, which had again formed an ulcer. Part of the diseased skin in the groin was destroyed with strong caustic. 
February 7. The sore in the groin had continued troublesome; a cluster of diseased glands formed its base, and there were several sinuses running in different directions in the cellular membrane around them.

The ulcer on the penis had again healed, with less hardness.

March 16. He felt sick and feverish. There were still numerous sinuses communicating with diseased glands in his groin, and the sores had a tendency to slough.

March 20. His febrile symptoms had subsided, and the sinuses were filling up. A sore bad extended to the depth of an inch and a half in the midst of a cluster of absorbent glands, close to the pubis, on the inside of the femoral artery. It had begun to look clean.

May 14. The groin had healed. The absorbent glands had subsided, and the hardness of the cicatrix of the sore on the penis had disappeared.

He was dismissed. 
2d. Sores which were followed by constitutional symptoms.

1. Papular eruptions.

\section{CASE XII.}

Thomas Campion was admitted into the Coldstream Hospital on the 3d of June, 1815, with a large, but not a very deep sore on the edge of the inner membrane of the prepuce. It had no disposition to granulate, and he had an open bubo in each groin. The sore had from his account been present a fortnight, and the buboes rather more than a week. The former was healed on the 14th of June, and on the 24th of that month, after feeling chilly and feverish for two or three days, an eruption of inflamed papulæ, like common lichen, appeared over his body, and about his forehead and neck. On the 28th the febrile symptoms had disappeared; but the eruption was very thick, and his left eye was inflamed. The inflammation did not appear to have extended to the internal tunics.

July 5. His eye was well ; the eruption had begun to fade, coming off in scurf. He had taken small doses of Epsom salts, and antimonial powder, since the first attack of the febrile symptoms. 
This was omitted, and decoction of bark and acid prescribed.

July 12. The eruption had nearly left him.

The buboes were both healed.

July 21. He was dismissed.

He returned four days afterwards, with violent pains in his limbs, increased at night. His ankles were swelled, and some fresh papulæ had appeared about his face and neck.

By the use of the warm bath and Dover's powder these symptoms were removed. He was again dismissed on the 21st of August, and has continued well since that time.

\section{CASE XIII.}

John Cartwright was admitted on the 12th of June, 1815, with an indolent sore close to the edge of the frænum, which he said had been present nearly two months. It was healed on the 25th of June, with some hardness and thickening, and he was dismissed.

August 20. He returned, covered with an erup- 
tion of lichen. This he said appeared about a week before, at which time he had severe pains in his thighs and legs. When admitted, the eruption was general over the face and body, and very thick about the arms and legs. The papulæ were small, and here and there filled with an opaque lymph, or puriform fluid; some were beginning to go off in scurf.

The eruption continued about three weeks, fresh papulæ occasionally appearing as the others went off. September 21st, nothing remained but a slight stain, and he was dismissed.

This man was of a consumptive habit, and has since been frequently a patient with pulmonary complaints. It was observed, that several of those who had been affected with sores were afterwards attacked with disorders in the chest. This, however, only took place in men, who were predisposed to such complaints. Scrophulous enlargements of the absorbent glands, particularly about the neck, were also frequently met with, and the tonsils often increased in size to a great degree. $\quad A$ young, drummer, who had been scrophulous in childhood, was affected with obstructions of the lachrymal ducts, and a collection of puriform fluid, in both lachrymal sacs, with great enlargement of the tonsils, five months after being cured of a very suspicious sore on the penis. Many of these disorders were no doubt totally unconnecte $d$ 
with the sores which happened to have preceded them; but there was some reason to suspect, that in a few of these cases an excitement was given to diseases, to which there had been a previous tendency, by the effects of the virus on the system.

\section{CASE XIV.}

John Dudman was admitted on the 16th of September, 1815, with a sore at the extremity of the prepuce, having thickened edges and some induration. He had also a bubo on his left groin.

The sore was healed in ten days, and the hardness of the cicatrix soon disappeared.

October 16. The bubo had subsided, and he was dismissed.

November 30. He was re-admitted; had felt chilly for several days, and was feverish at night. An eruption of lichen, or large distinct papulæ, had come out on every part of his body. Some of the papulæ were filled with a yellowish lymph or puriform fluid, and where this had oozed out, a thin scab was formed.

December 6. He was quite free from fever. The eruption was beginning to fade, going off in 
scurf. He was ordered decoction of sarsaparilla and nitrous acid.

December 13. His right testicle was enlarged; the swelling was uniform, and not very hard nor painful. A cold lotion was ordered.

December 26. The eruption had gone off, leaving a depressed stain of a brown colour, where each papula was. The testicle was reduced to its natural size. He was dismissed.

July 28, 1816. The stains left by the lichen were still perceptible.

\section{CASE XV.}

Thomas Wiles, forty years of age, was admitted into the Coldstream Hospital on the 30th of November, 1815, with a superficial ulcer of some days' standing, on the outer skin of the prepuce, neat its extremity. The glans could not be denuded, in consequence of the contracted state of the prepuce. He had been exposed to the risk of infection a short time before the sore appeared. It healed in three weeks; but the phymosis proving troublesome, the contracted ring at the end of the prepuce was cut off, and the inner membrane slit'open. He was dismissed on the 13th of 
January, 1816, being able to draw back the skin easily.

May 25, 1816. He was re-admitted. He said he had had an attack of fever in the beginning of February, whilst on furlough in Essex, and after it had continued for some days, an eruption came out on every part of his body. The surgeon who attended him thought at first it was small-pox. It was probably lichen, and the papulæ of a large size. They have large stains, which are slightly depressed, and becoming of a natural colour in their centres. The eruption, from his account, continued very thick about a week, and then went off in scurf.

On his admission he had enlarged absorbent glands about the angles of the lower jaw, was deaf, and had loss of appetite.

A bitter infusion, with a slight purgative, and diluted sulphuric acid, was prescribed.

On the 16th of June, after feeling chilly and feverish for two or three days, he was attacked with inflammation and tumefaction of the uvula and soft palate, extending along the palatine arches, and affecting both tonsils. This was soon followed by numerous aphthous ulcers. The disease extended to the epiglottis, and probably along the mucous membrane lining the glottis and larynx, vol. viri.

C C 
producing extreme hoarseness, violent pain under the pomum Adami, and an incessant irritation from collections of ropy mucus in the trachea. He was totally unable even to make an attempt to swallow for some days, and could not lie down for an alarm of suffocation, owing to the copious secretion of mucus. Blood was taken from his arm several times, and large blisters were applied to the throat. The blood was much cupped, and covered with a thick coat of buff. As these symptoms subsided, he had an attack of erysipelas of the face and neck, and was exceedingly reduced. He began to recover in the end of June and early part of July, but this was retarded by suppuration of several of the enlarged glands in his neck. These symptoms were probably not at all connected with the sore.

September 10. The abscesses in his neck were healed. He had taken bark and sulphuric acid for the last two months, and his health appeared re-established. The stains left by the eruption were fainter, the central part, which was of the natural colour, having gradually enlarged. Dismissed.

\section{CASE XVI.}

George Atkinson, of a scrophulous habit, twenty-eight years of age, on the 16th of December, 1815, whilst in hospital with pneumonia, was found 
to be affected with phymosis, attended with much dark-coloured discharge from under the prepuce. The sore could not be brought into view, but he said it was situated near the frænum; that it had been present nearly a month, and came four days after suspicious connection. He had an open bubo of a fortnight's standing in each groin. The phymosis was so far reduced on the 23d of December, that a deep foul sore was brought into view on the left side of the frænum, of the size of a silver penny, with hard and irregular edges. This was healed with much hardness on the 12th of January, 1816 , and on the 27 th of that month the buboes were well, and he was dismissed. In the beginning of April, whilst in hospital with pulmonary complaints, he had an attack of lichen. The eruption was similar to that in the former cases, but not so copious. The papulæ went off in scurf; fresh ones appearing from time to time. They left the usual stains.

In the end of the month he was attacked with measles. No fresh papulæ appeared from the period of the rash coming out.

This man had afterwards scrophulous abscesses, in the absorbent glands about the clavicles; with cough, and other symptoms of diseased lungs. These were no doubt quite unconnected with the sore. 


\section{CASE XVII.}

George Peace was admitted on the 21st of Fe. bruary, 1816, with an eruption of lichen over every part of his body. The papulæ were of a large size, very thickly, but equally diffused, and many of them filled with yellowish lymph. They appeared two days before, with pains in his shoulders, but without any distinct febrile symptoms. He had also a dark-coloured imflammation of the integuments covering each skin, a little above the ankles, which came on at the same time with the eruption.

He had had a sore on the internal prepuce in the end of November, 1815, which was cured in about a month, by a lotion he got from a surgeon in the country, without his using any mercury. This sore appeared about a week after suspicious connection, and has left slight thickening of the cicatrix, and a depression from loss of substance.

February 27. More of the papulæ have appeared on his forehead and face, and a great many over the lower extremities. Some matter had formed under the inflamed integuments of his right shin, which was let out. The boundaries of the abscess were not well defined. A probe could be passed to the periosteum, but the bone was not exposed. 
He has been in the warm bath, and has been well purged.

February 29. Fresh papulæ have continued to appear, and others have gone off in scurf. There was a zone of enlarged vessels round the cornea in each eye, but no irregularity of the iris.

Leeches were directed to be applied to thelower eyelids daily, and the opening medicines to be repeated.

March 2. The inflammation of the eyes was less.

March 13. The eruption was fading. The eyes were quite well.

He was ordered some decoction of bark and acid.

March 24. The eruption continued to fade, leaving only the usual stains. The sore on the skin was not healed.

April 21. His leg was well, and nothing rèmained but the stains of the eruption. He was dismissed.

August 23. The brown stains have been gene- 
rally getting fainter, and are not now very perceptible.

\section{CASE XVIII.}

Thomas Robins, twenty-eight years of age, was admitted on the 29th of June, 1816, with occasional pains in his shin-bones and knees, increased when he was warm in bed, but not so severe as to prevent sleep; a very copious eruption of lichen in large papulæ over every part of his body, and inflammation of both irides. There was a deep zone of vessels round the cornea; the pupils were irregular; and one or two large drops of lymph were effused in each iris on the margin of the pupil.

He said the pains in his limbs had been present about a month; that the eruption appeared about a week, and the inflammation of his eyes only three days previous to his admission; that he had had a similar eruption, though not nearly so copious, about three months before; and there were some stains on his forehead, which might be the remains of that attack.

He was a little hoarse, and had been so at different times during the winter; his tongue was much furred, but his general health was little affected. 
He had a chronic enlargement of some absorbent glands in each groin, and a considerable thickened cicatrix of a sore at the root of the penis. From his account, the sore had appeared about a year before; he thought it was a chancre, and cured it in about three weeks by means of some blue vitriol, without taking any mercury. The enlarged glands had been present about six months; and as he was confused in his story, it is not improbable the sore occurred at the same time.

He was ordered a brisk purgative, and afterwards a pill composed of two grains of calomel and half a grain of opium, to be taken three times a day.

July 3. The gums were affected. The inflammation of the eyes was subsiding, and the drops of lymph were nearly absorbed. The eruption was beginning to fade. Extract of belladonna was applied to the eyebrows, and the medicines continued.

July 6. The zone of red vessels had entirely disappeared. The pupils were acted on by the belladonna, and the adhesions of the irides were elongating. His mouth was very sore; he has taken the pills for eight days.

Some opening medicine was ordered, and the pills left off. 
July 11. The eruption was nearly gone, leaving the usual stain. His gums were much better, and the pupils were nearly circular, except when fully under the influence of belladonna.

July 29. He was dismissed, after being a few times in the warm bath.

The adhesions between the margin of the iris and capsule of the lens continued to be visible, at two or three points in each eye for some time; but by the 6th of October they were entirely absorbed, and no traces of the disease were left. The stains from the lichen were still very distinct.

He had afterwards over his shoulders and buttocks, and at one time on the soles of his feet, a few small vesicles, which came in clusters on a thickened and elevated patch of skin. They soon filled with a puriform fluid, and when they burst, left a thin scab. The thickened integument was of a dark-red colour, of an irregular form, and in general about an inch in its longest diameter. It continued for a long time, but produced no effect on his general health.

\section{CASE XIX.}

Elizabeth Payne, about twenty years of age, was 
admitted into the Saint James's Infirmary, on the 26th of October, 1816, with ardor uritr; purulent discharge from the vagina, and two or three superficial sores; with slightly thickened edges, on the inner surface of the vestibulum. She said these symptoms had been present a month.

November 9. She was covered over the face, scalp, and every part of the body and limbs; with an eruption of lichen. The papulæ were exceedingly minute, and so thick that not a particle of sound skin could be discovered, particularly about her forehead, which appeared puffed and swelled. Her tonsils were a little enlarged, but she had no pain in swallowing. The eruption began on the 7 th; and she had been feverish for a day or two pre. viously, with frequent rigors, headach, pain in her limbs, thirst, loss of appetite, and restlessness. The symptoms were still present; her tongue was white, and her pulse about 120. The discharge from the vagina continued, but the sores werehealed. Had taken small doses of salts and antimony for two days, which she was directed to continue.

November 23. The eruption continued, with the febrile symptoms. She complained of pain in the balls of the eyes, and over the eyebrows, and more red vessels were apparent on the conjunctiva than natural.

November 26. There was a distinct zone of ves. 
sels round each cornea, and the pain over the brow was increased. The eruption had begun to fade about the face, but was still very bright on every part of the body. She continued very feverish, and her countenance was much disfigured.

Saline diaphoretics were prescribed, and she was bled to twelve ounces. Leeches were directed to be applied daily to the lower eyelids.

December 4. The zone of vessels round each cornea was less bright, but the pupils were more irregular, and lymph was effused in the substance of the iris, and on the capsule of the lens. The extract of belladonna had been used to the eyebrows for some days. It was not deemed admissible to defer the use of mercury any longer. She was therefore directed to take two grains of calomel with half a grain of opium in the evening, and to repeat it afterwards twice a day.

The eruption on the face had nearly faded, coming off in scurf; on the body and limbs it was still very bright.

December 10. Her gums were affected, and the inflammation of the eyes were rapidly subsiding.

December 18. Her gums were very sore. The pills have been continued a fortnight. The pupils were irregular; but the eyes were in other respects 
well. Her febrile symptoms had subsided. The eruption on the body was fading, leaving behind minute brown stains slightly depressed. She was ordered to omit her pills, to have a solution of belladonna dropped into her eyes, and to take diluted sulphuric acid.

A few fresh papulæ of a larger size came out afterwards; but soon went off.

She used the warm bath occasionally, and in a short time recovered her flesh and looks. On the 29th of January, 1817, she was made an outpatient.

March 26. The stains of the lichen had nearly disappeared, and the adhesions of the iris could no longer be perceived in either of her eyes.

\section{May 6. She has had no relapse*.}

* I met with a similar form of eruption after a virulent gonorrhœa, uncombined with inflammation of the iris; and I saw a precisely similar case, both in respect to the disease of the skin and eyes, in a woman who was a patient of Mr. Brodie's in Saint George's Hospital. This woman said she had been affected with a discharge and ardor urinæ for some time, and believed she had also had some sores: An eruption of roseola annulata not unfrequently occurs in gonorrhœa, but I believe is to be attributed to the balsam of copaiba, and not to the disease, at least I never saw it except during the use of that remedy. It is sometimes accompanied with a good deal of fever. I saw a case last sum- 
Inflammation of the iris frequently occurs along with cutaneous eruptions, and is met with in almost every order and description of those diseases. Like them it is often excited by the venereal virus, under which circumstances it has been supposed that there is something peculiar in the appearances of the diseased membrane. The means of distinguishing between the simple and syphilitic forms of this inflammation have been pointed out by the late Mr. Saunders, and by Dr. Farre; but the diagnosis is not satisfactory. The means of cure are fortunately the same in both. Mercury is equally beneficial in the one as in the other, and is equally certain, if used at an early period *.

The good effects of that medicine cannot be more beautifully illustrated than in this disease. The deposition and organization of lymph is so rapid, that before the cure was effected by other means, (which it probably always might be) the powers of vision would often be permanently impaired. This is confirmed by the numerous cases of closed pupil and opaque capsule, which are met with from the neglect of the disease.

mer which had created considerable alarm, being mistaken for scarlatina. It was in a young officer of dragoons, who had been affected with scarlatina several years before.

* This most important fact is clearly explained in the last edition of Mr. Saunders's work, by Dr. Farre. 
To prevent any risk of this nature, I had recourse to a little mercury, whenever inflammation of the internal tunics of the eye was decidedly established. In several cases, along with different eruptions, there appeared a tendency to it, but it was checked by antiphlogistic remedies, except in the two last, before any lymph was effused; and in these the quantity of calomel which was given, could not be supposed to have produced a permanent cure, if they had been really venereal. This they probably were not. The sores in the last case were quite superficial, and the account of the primary symptoms in the other was very confused.

It appears that most of the papular eruptions followed ulcers which were not very deep, and which healed without much difficulty. Several of them had a thickened, but not a particularly indurated margin. This corresponds with the observations of Mr. Carmichael, to which I shall have occasion to refer afterwards. I could not, however, discover any decidedly uniform character in such sores; and the 16th case I should have considered as a well-marked instance of chancre. In it the man had pneumonia; and the excited state of his system, with the local effects of a phymosis, may account for the appearances of the sore. 
2. Constitutional Symptoms, differing from Papular Eruptions.

\section{CASE XX.}

John Lee was admitted into the Coldstream Hospital, on the 5th of September, 1815, with two foul sores, each of the size of a silver penny, on the internal prepuce, which had all the characters of chancres, and had been present seventeen days.

September 16. A bubo had appeared in his right groin.

October 30. The sores were healed, and the hardness of the prepuce was diminishing. The bubo was dispersed.

November 9. He had rheumatic pains in his shoulders and arms, and a dark mottled appearance of the skin over every part of his body. Some opening medicines were ordered.

November 25. The mottled appearance had begun to fade, and the rheumatism was less severe. Cicatrix of the sores natural.

December 15. The symptoms had disappeared. 


\section{CASE XXI.}

E. Hogg was admitted into the Coldstream Hospital, on the 13th of November, 1815, with a deep ulcer, with hard irregular margin, on the inner membrane of the prepuce. It was of the size of the diameter of a large split pea; and he had bubo in his right groin. The symptoms were of a few days' standing.

December 16. The sore was healed with a great deal of thickening and hardness. The bubo had suppurated, but the matter was beginning to be absorbed.

January 2d, 1816. A very irritable sore was again formed, from his having rubbed the cuticle off the cicatrix ten days ago. The whole prepuce was inflamed and swollen.

February 7. The sore had again healed. The hardness was like a piece of marble. The bubo had come forward, and had burst and healed. His skin had a dark mottled appearance over every part of his body.

February 28. By the use of a little mercurial ointment and camphor to the cicatrix, the hardness had a good deal diminished; but was still 
very considerable. The mottled skin was as before. He was dismissed.

April 16. The mottled appearance had entirely gone off. The hardness of the cicatrix remained.

September 21. The cicatrix was nearly natural.

\section{CASE XXII.}

William Carrier was admitted into the Coldstream Hospital, on the 23d of May, 1816, with a deep, foul sore by the edge of the corona glandis, near the frænum, one side of which was destroyed by it. The base and margin were much indurated and thickened, and the discharge was thin and acrid. The sore had been present a fortnight, and came four or five days after suspicious connection.

This sore continued for a long time exceedingly irritable, and was not healed till the 8th of August, and then with considerable hardness. A gland became affected in his left groin a few days after his admission, but was dispersed in about a month.

July 6. He observed some spots on his breast and loins, and in a day or two, his whole body was covered very thick with dark-brown patches, of an irregular form, and a little elevated, larger than the 
diameters of split peas, giving a mottled appearance to his skin. A few were visible on his forehead, about the roots of his hair, and behind his ears. The sore at the time this appeared was not giving much disturbance; he was thin, but his health was good.

July 17. He began to take sarsaparilla; the appearance of the eruption had not altered.

August 8. There were still some coppery-spots on his forehead, and about the roots of his hair; but those on the body were much fainter.

August 21. The sore had healed nearly a fortnight. The eruption was faint, and his health did not appear to suffer. His medicines were omitted, and he was dismissed.

October 6. A dark-coloured eruption, slightly elevated, had again become more distinct on his back and shoulders.

November 24. His tonsils were enlarged, and looked as if covered with an additional layer of lymph.

February 9, 1817. The same appearance of the tonsils continued. The eruption had disappeared.

February 23. The tonsils were nearly natural. vol. VIII.

D D 
His health has been uninterruptedly good, since the pain and irritation of the sore subsided.

\section{CASE XXIII.}

William Bowdler, twenty years of age, was admitted into the Saint James's Infirmary, on the 24th of May, 1816, with a deep sloughy chancre of six weeks' standing, which had destroyed a portion of the corona glandis. I gave him five grains of the common mercurial pill twice a day, after he had been in the infirmary for a fortnight; it was continued only for four days. His gums were rendered a little turgid.

June 25. The sore was healed with much hardness, and an enlarged gland, which had appeared in each groin a few days after his admission, was dispersed.

An eruption of a coppery colour, in flat spots a little elevated, and not regularly circular, about the size of large split peas, had appeared on his back and shoulders.

July 1. As his health was not suffering, I made him an out-patient.

July 23. He did not return till this day, since 
the 1st. He was covered over every part of the body with a pale coppery eruption, in patches of the size of a silver penny, rough on the surface from slight scurf of the cuticle, but not elevated. His ankles were swollen, and he had pains in his shoulders and limbs, when he walked; but slept well at night. Both tonsils were much enlarged, but not painful. His pulse was 108 ; his health was good. The cicatrix of the chancre was still very hard.

I ordered him a drachm of powder of sarsaparilla three times a day; but he did not take it with any regularity after the first week.

September 10. He was perfectly free from the eruption. His tonsils were less, but still above their natural size.

May 9th, 1817. This man has been seen occasionally during the winter and spring, and has had no return of his complaints. The hardness of the cicatrix gradually went off.

July, 1817. He returned with some warts on the cicatrix : his health perfectly good.

\section{CASE XXIV.}

David Philips was admitted into the Coldstream

D 2 
Hospital, on the 12th of December, 1815, with a sore having the common appearances of chancre. It was healed with much hardness and thickening on the 13th of January following, and he was dismissed.

April 16. His health has been good, and there has been no appearance of any cutaneous affection, but his hair has come off in large patches, particularly about the back of his head.

July, 28. For the last month his hair has begun ta grow again as usual, and is now of the natural thickness.

Another case nearly similar to this occurred; whether the symptom was accidental, or connected with the sore, is not easily determined.

\section{$C A S E X X V$.}

William Simmonds, a corporal, was admitted into the Coldstream Hospital, on the 24th of Janu. ary, 1816, with a deep, foul, irritable sore, on the right side of the internal membrane of the prepuce, with hard, irregular edges, and considerable thickening. 'It had been present eight or ten days, and followed a suspicious connection. It had produced a bubo in his right groin, which was 
as large as a hen's egg. The sore was not healed till the middle of March, and then with a very hard cicatrix. The bubo also was troublesome; it suppurated, and burst in different points over diseased glands.

March 24. A portion of diseased skin in the groin had sloughed away, and left a clean sore. His right tonsil was a little enlarged, and he had an eruption of pale coppery spots over his shoulders, neck, and forehead. The spots were of an irregular form, and scarcely any where elevated. He had felt feverish and out of sorts previous to the appearance of these symptoms.

April 1. The eruption was more general over his body. His right tonsil was much swollen, and had a great deal of pain in his throat at night. His pulse was above 110. He was ordered a pint of decoction of sarsaparilla, with a dram of extract daily.

May 1. His health was much improved; the eruption had disappeared, and his tonsil was much smaller, and free from pain. The hardness of the cicatrix of the chancre was still very considerable.

His medicines were discontinued, and he was dismissed.

June 1. He was re-admitted, with pains in his 
loins and knees, increased at night, and prevent. ing sleep.

July 10. A small tumor, of an oblong shape and of the size of a walnut, had appeared in the middle of his left leg, immediately between the inner margin of the soleus muscle and the bone. It was close to the latter, and was extremely tender to the touch; but he had no pain or pressure over the anterior surface of the tibia. To use the warm bath every night.

July 12. The pain in the tumor was less. His gums were spongy, as if from the action of mercury, but he denied having taken any medicines not prescribed for him; and he was the first to call the attention to the tenderness of the gums.

A decoction of bark with acid was prescribed.

July 24. The swelling was nearly gone; the pains in his limbs had left him, and his gums were quite well. Has discontinued the warm bath for several days.

July 29. The tumor was quite dispersed, and he appeared in good health. Dismissed.

He was watched very carefully afterwards, lest he should be using any mercurial preparation, but nothing appeared to warrant such a suspicion. On 
the 29th of December he was discharged the regiment, and he promised to acquaint me if his disorder returned. I have not since heard of him.

\section{CASE XXVI.}

Thomas Thelbay was admitted into the Coldstream Hospital, on the 16th of March, 1816, with a small, deep, circular sore, with hard and irregular edges, immediately behind the corona glandis, and two small sores on the outer edge of the prepuce. He had also some degree of phymosis, a purulent discharge from the urethra, and a gland was enlarged to the size of a hazle nut in his right groin.

May 8. The sore behind the corona was.beginning to granulate. It had proved very troublesome, several deep sloughs having formed in it at different periods. A pustular eruption had appeared on his body and limbs. The pustules were very small, not much larger than pin's heads, and were on slightly elevated and dark-red bases. His tonsils were a good deal enlarged, especially the left, and there was some ulceration in the back part of the pharynx. The eruption had commenced two days before; he had some pain in his loins, but no distinct febrile symptoms. 
He was ordered to use the warm bath.

May 15. The eruption had extended to his forehead, chin, ears, mouth and neck. His right ankle swelled at night, and he complained of pain in the upper part of the tendo Achillis. Numerous tubercles could be perceived over all the inner part of his right leg. They were situated under the integuments, felt perfectly moveable, and were about half the size of garden peas. His tonsils, and the absorbent glands about the angles of the jaw, were much enlarged. He was ordered to repeat the warm bath every other night, and to take a pint of decoction of sarsaparilla daily.

May 20. The conjunctiva of the left eye was very vascular, and a zone of red vessels surrounded the cornea. He had pain in the globe. The eruption was not altered. The sarsaparilla was omitted, and some opening physic prescribed. Six leeches were applied to the lower eye-lid and temple, and a cold lotion was afterwards kept to the eye.

May 26. Three leeches have been applied daily since the 20th, and the inflammation of the eye has entirely subsided. Many of the pustules have burst and formed a thin crust, and fresh ones have continued to come out.

June 1. The eruption covered every part of his 
body. The pustules had increased in size; but were still a good deal smaller, and were of a darker. colour than small-pox. On the face they were beginning generally to scab. The following medicine was prescribed.

R Infus. Gent. comp. 亏̌vi. Sennæ 3iss.

Liq. Potassæ 3 ss m. f. mist. capt. ऊiss ter die.

June 3. The eruption had begun to scab on the body. His pulse was quick; he was a good deal reduced, and his ankles swelled when he took exercise.

June 15. A fresh crop of pustules came out on every part of his body, of a much brighter red than the last.

His pulse was nearly 120 , but he slept well, and his appetite was good.

He was ordered a pint of decoction of sarsaparilla daily.

July 1. The eruption was nearly gone. It had left dark-brown spots wherever the pustules had been. Those on the legs were of a large size, as the pustules there had been situated on broad bases. The tubercles under the skin were much diminished. 
His ankles swelled at night.

July 20. He has continued his sarsaparilla with the addition of some extract, and has used a warm salt-water foot-bath. His health was much improved; his tonsils continued as large as plover's eggs, but gave no pain. He was dismissed.

August 12. He was re-admitted with febrile symptoms and a redness over the skin; his tonsils were much as before, and there was a mass of enlarged absorbent glands in his neck about the upper part of each mastoid muscle.

August 14. Pustules, not situated as before on inflamed bases, but immediately filling with a puriform fluid, had again come out on his back and shoulders, and a few on his forehead, on the hairy scalp, and about the pubes. On inverting the upper eyelids, which were turgid, numerous small ones were discovered on the conjunctiva lining them.

September 6. The eruption had gradually disappeared, and the spots it left were fainter than formerly. Those on his legs were of a dark copper colour, were depressed below the neighbouring skin, and occasionally lighter coloured in their centres. His tonsils were large, but his looks were much improved. He was dismissed after taking bark and acid for a fortnight. 
A similar eruption appeared on the $2 d$ of October. It was fainter, with less disturbance than any of the preceding ones, and seemed to shew that the constitution was gradually overcoming the force of the poison. It was entirely gone on the 9th of November. His legs continued to swell for some time, and he had a few ill-conditioned ulcers on ithe ankles, such as generally occur in œdematous limbs. The eruption did not return afterwards, and his general health gradually improved.

\section{CASE XXVII.}

John Macdermot, a drummer, sixteen years of age, was admitted into the Coldstream Hospital, on the 28th of June, 1816, with two sores, which he said had been present five or six weeks, and came a few days after a suspicious connection. They were of a considerable size, and had the common characters of chancre. They were healed with a good deal of hardness on the 26th of July. He had also a bubo in his right groin, which subsided.

July 29. An eruption had come out over his legs and arms, and slightly over his shoulders. It consisted of dark brown spots, not elevated, but a little scurfy on their surface; varying from the size of a sixpence, to that of the diameter of a large split- 
pea. The largest were of the faintest colour. He had also inflammation of the periosteum of the right shin-bone, near its middle. It was a little swelled, and very painful to the touch.

Both his tonsils were swelled, and slightly ulcerated; he complained of noise in his ears when in bed, but he slept well, and his generalthealth was not affected.

He was ordered some opening medicines, an astringent gargle, and a few grains of compound ipecacuanha powder at night.

August 3. His throat was easier, and the periosteum less tender.

He was ordered some bark and acid.

August 23. The node had disappeared, and the eruption was extremely faint. His tonsils were still much enlarged, but the ulceration had healed. They had a lobulated appearance; the lobes resembled the fringed margin of the comb of a cock, but thicker and larger.

He left off his bark, and took twenty-five drops of diluted sulphuric acid three times a day.

September 6. His health continued good: the eruption was fainter, and he was dismissed. 
January 20, 1817. The eruption was no longer perceptible, but the tonsils did not assume their natural appearance till towards the end of $\mathrm{Fe}$ bruary.

\section{CASE XXVIII.}

John Atkinson was admitted into the Coldstream Hospital, on the 4th of July, 1816, with a circular sore on the outer skin of the prepuce, with the common characters of chancre. It was healed with much hardness on the $2 \mathrm{~d}$ of September.

October 6. Aphthous ulcerations had appeared on both tonsils, unattended with much pain.

October 14. Several whitish patches of thickened cuticle were seen on the insides of his lips, of a few lines diameter, giving the appearance as if a layer of lymph was deposited there. There was an angry ulceration running along the upper margin of the gums, by the fangs of the teeth of the lower jaw. The tonsils also looked as if covered with a coat of ill organized lymph, but the aphthous ulcers had healed. He had a pricking sensation in swallowing, which hardly amounted to pain. These symptoms continued until the end of January, and then gradually disappeared.

Many other cases might be given, differing in 
some respects from those above detailed, but 1 shall only briefly refer to a few of these.

One man had enlarged tonsils, and an eruption of bright flat coppery spots, appearing among the roots of his hair and eyebrows, and on his back and shoulders. These symptoms attacked him about six weeks after a very angry sore, and the eruption continued for more than five months. His hair came off wherever the blotches had been situated. The tonsils did not recover their natural appearance for two months longer. He had much febrile disturbance at different periods, particularly on the first approach of the secondary symptoms.

Another was covered over the breast and back, with a pale coppery eruption in extremely small spots, not exceeding half a line in diameter. Many of these were grouped together into different figures, of circles, horse-shoes, \&c. They were scarcely at all elevated. They appeared four months after the primary symptoms, and produced no disturbance whatever of the general health. They continued for nearly four months.

A third had mottled skin, great emaciation, violent pains in his shins and elbows, and œdema of the legs. These followed a most painful spreading chancre, which was as difficult to heal, as any I had met with. They came on at different periods; 
the mottled skin about six weeks after the appearance of the sore, and whilst it was still very irritable, and the pains in the limbs in about double that period.

None of these constitutional symptoms proved tedious.

A gentleman had a mottled skin two months after a sore, and, in about ten months more, had psoriasis of the scrotum and penis. The last symptom continued for more than five months; and got well whilst he was using the salt-water bath.

Another had mottled skin and pustules over the scalp, two months after a sore, which began like a pimple, and ulcerated at its apex, healing in about five weeks, and leaving an elevated smooth darkred cicatrix, tender to the touch. He had afterwards ulceration of both tonsils, which recurred frequently during several months. The ulcers had an irregular margin, and both tonsils had an appearance, as if covered with a thin coat of lymph. They were at times very painful, particularly at night. The cicatrix was more inflamed and painful at the time these symptoms came on. He experienced much relief from having the ulcers in his throat touched daily with a strong solution of lunar caustic. He took only purgative medicines, and the symptoms went off in about seven 
months. This case was seen occasionally by Mr. Brodie.

1

I have already trespassed much too far on the time of the Society, and shall therefore offer but a very few concluding remarks.

The impossibility of effecting a cure of syphilis, except by the exhibition of mercury, has for so long a period been admitted as an established principle, and has been so generally adopted as a leading characteristic of the disease, that I cannot expect the facts and cases which $I$ have related, to do more than lead to a further investigation of -so intricate and important a question. If that principle is erroneous, new views of the subject, of material consequence, will evidently be opened to us; and part of the difficulties which have been met with in distinguishing between.syphilis and some other diseases, will naturally be accounted for, by the mistaken grounds on which a distinction has often been looked for, where none in reality existed.

That the diseases which are commonly communicated by sexual intercourse, do not all arise from one peculiar poison, is an opinion which can hardly be doubted. Long before syphilis appeared, some of them were frequently met with, and Mr. Peaison thinks, that, in addition to those formerly known, new forms of disease have occasionally 
arisen, "which are succeeded by a regular series of symptoms nearly resembling the progress of Lues venerea *" How far the variety which we meet with, in the symptoms of venereal cases, is to be attributed to different poisons; or how far the symptoms of the same poison may be modified, and altered by constitution, climate, and habits of life, is as yet merely hypothesis. We have seldom an opportunity of tracing different cases to the same source of infection, so as to ascertain how nearly, under such circumstances, they would correspond. Inoculation, if admissible, would throw much light on this interesting question.

Mr. Carmichael has attempted to arrange these diseases under distinct heads, and thinks he can point out, satisfactorily, several kinds of venereal sores well characterized, each of which he supposes to arise from a different poison, and to be followed by its peculiar constitutional symptoms. He has found all these curable without mercury, except what he calls the syphilitic chancre, and the secondary symptoms which arise from it: viz. the excavated ulcer of the tonsils, the scaly eruption on the skin, and some peculiar affections of the bones. It would certainly be an important improvement in surgery, if such an arrangement could be made, and such a degree of accuracy attained; but the appearances

* Vide Observations on the effects of various articles of the Materia Medica in the cure of lues venerea. Second Edition. Introduction, p. 53.

voL. VIII. E E 
of sores can seldom be relied on, in part of such vascular structure, and in the midst of sebaceous glands. Peculiarity of constitution must also be taken into account. I recollect many years ago, a healthy young man, who was affected with a decidedly sloughing sore on the penis, in consequence of a suspicious connection. It was not attended with any constitutional disturbance, and yielded readily to mercury. He twice afterwards, at a very considerable interval, had a fresh infection, and the sores each time had precisely the same character with the first. This is no uncommon occurrence, and it is not probable, that the sloughing and appearance of the sores arose from the peculiarity of the poison, when it is so easily explained by the effects of the constitution. But although the character of the primary sore, may, like that of any other ulcer, be modified by a variety of causes, it is not easy to suppose that these can also account for the great difference in the secondary symptoms. Can the influence of constitution, alone, for instance, enable the same poison to produce a papular eruption in one individual, and a pustular, an exanthematous, or a scaly eruption, in another? This at least does not happen in any other disease arising from a morbific poison ; we may hope therefore, although the inquiry is a difficult one, that some useful distinctions with respect to the venereal poisons will in time be made. Mr. Carmichael is no doubt continuing the investigation, and his opportunities of practice are extensive. 
I already stated that he considers the true syphilitic chancre, and the symptoms which arise from it, as in every instance absolutely requiring mercury. This appears to me incorrect. I have certainly cured, without that specific, ulcers which had a decidedly marked induration of the margins and bases, by which the syphilitic chancre, according to him, is easily distinguished. It is, also, obvious to every surgeon of experience, that the excavated ulcer of the tonsils, as described by $\mathrm{Mr}$. Hunter, is not, as Mr. Carmichael seems to think, a peculiar symptom of the presence of the syphilitic virus. I have repeatedly seen it, as well as the scaly blotch, in cases where mercury had been fieely employed for the primary sores, and in which I considered the virus as eradicated, and both have disappeared under the use of sarsaparilla. It may be added, that the sternum and clavicle, which $\mathrm{Mr}$. Carmichael mentions, as two of the bones most liable to the disease, are by many surgeons not supposed to be ever affected by true syphilis*.

The cases of which I have given a detail, will be sufficient to shew the common progress of the symptoms, in the diseases which I met with during the course of this inquiry. It may surely be assert-

- In his most valuable Lectures on this disease, Mr. Pearson stated, in describing the bones commonly attacked by syphilis, that the sternum, clavicles, and ribs, were frequently affected by cachexia syphiloidea; 'but . he added, I think, that he had never seen venereal nodes in them. 
ed that, in many of them, the sores had all the appearances usually considered as characteristic of true chancre. Some were probably of a different nature, but the majority would, I believe, have been submitted to a course of mercury, even by the most cautious practitioners. Without including many slighter ulcerations, and those of which I lost sight, immediately after their cure, I have, during the last two years, treated on the same system more than one hundred and twenty cases, where I have been able to ascertain that my patients were in perfect health for many months afterwards, or where they have returned to me with secondary symptoms, similar to those already described. Upon an average, one out of every three of the sores thus treated, was followed by some form or other of constitutional affection; this was in most instances mild, and sometimes so slight, that it would have escaped notice, if it had not been carefully sought for.

The constitutional symptoms were evidently not such as could be regarded as venereal, if we give credit to the commonly received ideas on the subject. Caries of the bones, and some of the least equivocal symptoms, did not occur. In no instance was there that uniform progress, with unrelenting fury, from one order of symptoms and parts affected to another, which is considered as an essential characteristic of true syphilis. Even each individual symptom of that disease has been declared by 
some to be regularly progressive, and never to be checked except by the influence of mercury. $\mathbf{M r}$. Abernethy inquired, he tells us, of the best surgeons in London, whether constitutional symptoms of syphilis do ever spontaneously amend? No one decidedly replied in the affirmative; and Mr. Hunter considered the disappearance of any symptom where mercury had not been employed, as a proof of its not being syphilitic.

I have purposely avoided introducing at present any instances of those more aggravated consitutional diseases, which are of such frequent occurrence, where the previous history is confused by the injudicious use of mercury. In these we are often at a loss, whether to attribute the symptoms to a different disease, induced by the remedy, or to consider them as the effects of the original poison, which that remedy had not completely subdued. It is astonishing how widely the opinions of medical men differ respecting these; but amongst cautious practitioners a very small proportion of them is submitted to a course of mercury, in comparison with what would formerly have been supposed to require that treatment.

The cachexia syphiloidea, or pseudo-syphilis, to which a great many of these cases are referred by the surgeons of this country, is often a most obstinate and formidable disease. It was not distinguished from syphilis till of late years, and pro- 
bably some genuine cases of the latter have been treated and cured under such a denomination. Mercury, if carried to any extent, produces in it most pernicious effects ; indeed the cachexia syphiloidea is rarely met with except where that medicine has been freely employed, which is therefore considered as one of its exciting causes. It would appear from a remark of Dr. Scott's, however, that that cause is not alone sufficient to give rise to it. He states, "that during the whole of his residence in India, where mercury is so commonly, so largely, and sometimes so injudiciously given for affections of the liver, he never knew a single instance of this new disease having arisen where syphilis was certainly out of the question."

Like syphilis, the cachexia syphiloidea appears to be produced by some absorbed poison, and frequently follows different sores. I met with a wellmarked case of it lately after a very painful and ill-conditioned sore on the finger, and another which followed an ulcer on the lip. It has never been satisfactorily ascertained whether such sores have the power of secreting a matter which can produce the same disease in others.

There seems however no doubt, that in some of its stages the disease itself is contagious. I have known three instances of husbands having communicated it to their wives; and in two of these, I was not able to ascertain that there had been any 
sore after marriage, from whence inoculation could have taken place*. The same remark, with respect to our ignorance of the means of infection, applies to the disease with which infants are frequently affected shortly after birth, characterized by coppery spots, emaciation, and other symptoms, and supposed to be the effect of the virus of syphilis. We commonly find in such cases that one of the parents or the nurse have had some venereal or syphiloid disease at no very distant period; but the precise mode of infection can seldom be ascertained.

The modification which the symptoms of syphilis undergo, from the injudicious use of mercury, so conducted as to fail of effecting a cure, is an inquiry of considerable interest, and would, if properly conducted, throw a great deal of light on the history of the disease. It is now generally admitted that the majority, and certainly by far the most serious diseases of the bones, as well as many other most distressing symptoms which are met with in all these diseases, are to be attributed to the injudicious or excessive use of that remedy. Most lamentable cases of this description are still by no means uncommon; and when we reflect on these, and on the horrible excess in which mercury was universally employed, two or three centuries ago, we shall no longer wonder at the alarm then enter-

* Two cases of this nature are given by $\mathrm{Mr}$. Abernethy, in his Essay on Diseases resembling Syphilis. 
424 ON THE TREATMENT OF SYPHILIS.

tained of the disease, which was assumed to be fatal in itself, and was too often rendered so, by the means attempted for its relief.

On the subject of all these diseases, much further information is wanting, and this can only be obtained by an accurate attention to facts, which their frequent occurrence gives every one an opportunity of observing ; and by founding our opinions on a careful induction from these, and not on the vague theories which have hitherto so generally been adopted. If new forms of disease have arisen, or if syphilis be itself modified, both which doctrines some are inclined to maintain; these form only stronger arguments for clearing our minds of all preconceived ideas which are not found to bear the test of experience, and carefully investigating the symptoms and treatment of those diseases which now occur. I should be more inclined to suspect, from the extreme contradictions at all times to be discovered in our histories of syphilis, that the descriptions of it have been totally erroneous, even to the very circumstances and period of its origin, than that any such change has taken place. 